MEASUREMENTS OF RADIATIVE HEATING ON SHARP CONES

by

DALE L. COMPTON and DAVID M. COOPER

National Aeronautics and Space Administration

Moffett Field, California

AIAA Paper

No. 64-252

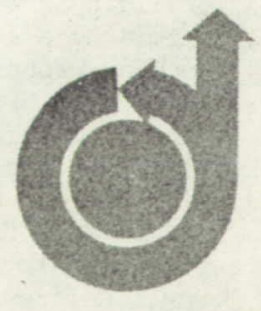

First publication rights reserved by American Institute of Aeronautics and Astronautics, 1290 Sixth Avenue, New York, N. Y. 10019. Abstracts may be published without permission if credit is given tó author and to AIAA. (Price-AIAA Member 50c, Non-Member \$1.00). 


\section{MEASUREMEITSS OF RADIATIVE HEATING ON SHARP CONES}

By Dale L. Compton and David M. Cooper

Research Sclentists, NASA, Ames Research Center, Moffett Field, California

\section{Summary}

Measurements of total radiative heat load have been made on $30^{\circ}$ half-angle, $1-\mathrm{cm}$ base diameter, circular cones in free flight. Models were gunlaunched into air in the prototype of the Ames Hypervelocity Free-Flight Facility with a nominal velocity of $7 \mathrm{~km} / \mathrm{sec}$. Free-stream pressure was the primary test variable and was varled from 2 to $200 \mathrm{~mm} \mathrm{Hg}$. Secondary test variables were angle of attack and model material. Tests were performed at seven pressures on ablating polyethylene models, at four pressures on ablating polyformaldehyde models, and at two pressures on nonablating metal models. The radiation from the shock layer was measured with several broadband radiometers consisting of S-5 spectral response photomultipliers. In addition, the shock-layer radiation was spectrally resolved into 12 components in the $0.22-1.15$ micron range with photomultiplier, optical filter radiometers.

Predictions of the total radiation were made for both equilibrium and nonequilibrium conditions in the shock layer. The equilibrium radiative intensities were taken from the work of Kivel and Bailey. The nonequilibrium radiative intensities were taken from the measurements of nonequilibrium radiation of Page and Amold combined with the radiative relaxation distance measurements of Teare, Georgiev, and Allen. Radiative intensities were computed along streamlines in the conical shock layer, and integrations of the local radiative intensities were then performed to obtain the total radiative heat load.

Results from the tests show that the radiant heat load to cones is much smaller than that to blunt bodies at comparable free-stream conditions, thus supporting the conclusions of Allen, Seiff, and Winovich.

Radiant power measured at the same free-stream conditions by the broadband radiometers was found to depeind on model material. The radiation from the shock layer of the ablating polyethylene models was always higher than that from nonablating metal and ablating polyformaldehyde models. The addi- . tional radiation on the polyethylene models is the emission from heated products of ablation in the boundary layer, as shown by comparisons of spectra for polyethylene with those for metal. Spectra from the polyformaldehyde models showed that ablation-products radiation was not present in the spectral range of the broadband detectors. In addition, good agreement was found between the broadband measurements on metal models and those on polyformaldehyde models, indicating that for the polyformaldehyde models the radiant emission in the S-5 spectral response range was primarily from air.

Comparison of the data from the metal and polyformaldehyde models with the predictions of radiation for air shows reasonable agreement. At the highest test densities, the measured radiation is approaching the equilibrium prediction, while at lower densities the measurements are substantially above the equilibrium prediction and agree

reasonably well with the prediction for combined equilibrium and nonequilibrium radiation.

\section{Introduction}

For entries into the earth's atmosphere at velocities well in excess of escape velocity, Allen, Seiff, and Winovich (ref. 1) have shown that the use of sharp entry bodies, such as pointed cones, will reduce the total aerodynamic heating. This is true since the radiative heating which dominates heat transfer to blunt bodies at these high velocities is reduced to a manageable level on cones by virtue of the relatively low gas temperatures behind the swept shock wave, and the reduction in radiative heating more than compensates for the associated increase in convective heating. To help verify these conclusions, experimental information is required on radiative heat transfer to cones. In addition, methods are required for the computation of radiative heat load which will account for both equilibrium and nonequilibrium radiation in conical flow fields. One purpose of the present paper is to present the results of free-flight range meas urements of radiative heat load to conical models. A second purpose is to develop a method of predicting the radiative heat load and to compare the measurements of radiation with the predictions.

A

$\mathrm{E}$

$\mathrm{E}_{\mathrm{EQ}}$

$\mathrm{E}_{\mathrm{P}}$

k

ᄂ

$P_{\infty}$

$\mathrm{V}_{E Q}$

$\mathrm{V}_{\infty}$ W

$\mathrm{W}_{\mathrm{EQ}}$

$\mathrm{x}, \mathrm{y}$

$\mathrm{x}_{1}, \mathrm{x}_{2}, \mathrm{x}_{3}$

$\mathrm{y}_{\mathrm{p}}$

$\alpha$

\author{
Symbols \\ area, $\mathrm{cm}^{2}$ \\ radiative intensity, watts $/ \mathrm{cm}^{3}$ \\ equilibrium radiative intensity, \\ watts $/ \mathrm{cm}^{3}$ \\ peak nonequilibrium radiative intensity, \\ watts $/ \mathrm{cm}^{3}$ \\ exponential radiative intensity decay \\ factor, $\mathrm{cm}^{-1}$ \\ cone slant length, cm \\ free-stream pressure, $\mathrm{mm} \mathrm{Hg}$ \\ equilibrium radiating volume, $\mathrm{cm}^{3}$ \\ free-stream velocity, $\mathrm{km} / \mathrm{sec}$ \\ radiant power, watts \\ equilibrium radiant power, watts \\ Cartesian coordinates \\ coordinates defined in sketch (c) \\ distance defined in Eq. (B5) \\ angle of attack, deg \\ angle between the cone surface and \\ equilibrium conical shock wave, deg
}


op radiative excitation distance, cm

$\delta_{r}$ distance required for radiation to decay to $1.1 \mathrm{E} \mathrm{EQ}$, cm

$\theta_{c}$ cone half-angle, deg

$\sigma_{\mathrm{v}} \quad$ viewing angle defined in sketch (c)

$\varphi, \eta$ angles defined in sketch (b)

$x$ angle-of-attack correction factor

\section{Superscripts}

* distances and exponentlal radiative decay factors along a streamline behind a normal shock wave in one-dimensional flow

- distances along a streamline in a conical shock layer

\section{Experiments}

\section{Facility}

Models were launched from a light-gas gun into the prototype of the Ames Hypervelocity Free-Flight Facility, which for this investigation, was operated without its countercurrent air stream as an enclosed ballistic range. A schematic view of the facility is shown in Fig. 1 . The model launcher is a caliber 0.50 , deformable-piston, light-gas gun. Models $f l y$ through an instrumented test section equipped with 1 il spark shadowgraph stations spaced at 4-foot intervals along its length. An enlarged view of part of the test section is shown in the inset to FIg. 1. Time-of-flight intervals between stations are recorded with electronic chronographs. The time-distance history and the angle-of-attack history of the model are extracted from the shadowgraph pictures and chronograph records. Freestream properties are determined from measurements of the pressure and temperature in the tunnel just prior to launching the model.

Radiometers

Radiation from the shock layer of the models was measured with six identical broadband radiometers, deplcted schematically in Figs. 1 and 2 ." Each radiometer consisted of an S-5 spectralresponse photomultiplier (type RCA IP28). These tubes were mounted in housings on the test section walls with slits which limited their flelds of view, and were placed in pairs on orthogonal viewing lines. Thus, each pair of instruments made simultaneous measurements on the model from different viewing directions. The spectral range covered by the broadband radiometers was from 0.2 to 0.6 micron between the 10 percent of maximum response points.

Radiation from the shock layer was resolved into 12 spectral components by another set of radiometers also shown schematically in Fig. 1. The spectral range covered was from 0.22 to 1.15 microns. Each radiometer consisted of a photomultiplier and a narrow-pass optical filter. The 12 channels of this intrument were mounted together and viewed the model from nearly the same direction. Similar radiometers are described in more detail in reference 2 which also describes the methods used to calibrate the radiometers for their absolute radiant sensitivity. Output signals from the radiometers were displayed on oscilloscopes and photographed.

Models and Sabots

The models used for these tests were $30^{\circ}$ halfangle, right-circular cones with a base diameter of $1.02 \mathrm{~cm}$. The models were machined from several different materials. Plastic models, which ablated during flight, were machined from polyethylene and from polyformaldehyde. Metal models, which did not begin to ablate before passing the radiometers, were constructed from aluminum with copper tips. A sabot machined from polycarbonate plastic was used to fit each model to the launch tube of the gun. A sketch of a model and sabot is shown in Fig. 3 .

Test Conditions

The nominal test velocity was $7.0 \mathrm{~km} / \mathrm{sec}$. However, the actual test velocities varied from 5.7 to $7.3 \mathrm{~km} / \mathrm{sec}$. Polyethylene models were tested at free-stream pressures of $2,5,10,20,50,100$, and $200 \mathrm{~mm} \mathrm{Hg}$. Polyformaldehyde models were tested at pressures of $20,50,100$, and $200 \mathrm{~mm} \mathrm{Hg}$, and metal models were tested at pressures of 10 and $20 \mathrm{~mm} \mathrm{Hg}$. Free-stream temperature was room temperature for all tẹsts.

Reduction of Broadband Radiometer Data

The final output desired from the broadband radiometer data was the number of watts emitted by the conical shock layer of a model flying at zero angle of attack and at a velocity of $7 \mathrm{~km} / \mathrm{sec}$. To obtain this information, the radiometer data were reduced in several steps.

1. The output of the radiometer was multiplied by its calibration constant which was adjusted by the inverse square law to correspond to the distance from the radiometer to the model.

2. A correction was then applied to account for the difference between the spectral energy distribution of the air radiation in the shock layer of the model and the spectral sensitivity of the photomultiplier. The radiometer responded to approximately 80 percent of the air radiation.

3. A geometric correction was applied to account for the radiation in the part of the shock layer hidden from the photomultiplier. At zero angle of attack, the radiometer can see approximately 70 percent of the shock-layer volume. This shadowing effect is relatively insensitive to angle of attack.

4. A correction was applied to account for angle of attack of the model. On cones, shocklayer temperature and, thus, radiation are sensitive to angle of attack. To make the correction the temperature and density in the conical shock layer at angle of attack were assumed to be those for the appropriate tangent cone. The predictions of reference 3 were then used to obtain the equilibrium radiative intensity distributions around the cone. These distributions were then integrated 
over the portion of the model visible to the photomultiplier and divided by the zero angle-of-attack value to obtain a correction factor. Corresponding computations were made for nonequilibrium radiation in the shock layer. The methods used are presented in more detail in appendix A. The success of the corrections can be inferred from their effect on the data and is discussed in the section on results and discussion.

5. A correction was made to shift the data to a standard velocity of $7 \mathrm{~km} / \mathrm{sec}$. Each data point was multiplied by the ratio $\mathrm{E}_{\mathrm{EQ}_{7}} / \mathrm{E}_{\mathrm{EQ}}$ where $\mathrm{E}_{\mathrm{EQ}}$ is the equilibrium radiant intensity at the test condition and $E_{\mathrm{EQ}_{7}}$ is the equilibrium intensity at the same density but for a free-stream velocity of $7 \mathrm{~km} / \mathrm{sec}$. The equilibrium intensities were again taken from the predictions of reference 3 .

In sumary, it was necessary to correct the raw data for viewing distance, spectral sensitivity of the photomultiplier, geometry of the shock layer, angle of attack, and slight differences in test velocity to obtain the final data.

\section{Reduction of Spectral Data}

Each output from the 12-channel radiometer was reduced to watts emitted per micron of wavelength by the method described in reference 2. No attempt was made to correct these data for angle of attack, or to compensate for test velocities different from $7 \mathrm{~km} / \mathrm{sec}$. The principal use for the spectral information was to discover which of the broadband radiometer data were being influenced by the presence of radiation from heated products of ablation in the boundary layer. The influence of this extraneous source of radiation on the data will be considered in the section on results and discussion.

\section{Radiation Predictions}

Predictions of the radiation to be expected from the conical shock layer for the test conditions were made by the following methods.

\section{Equilibrium Radiation}

To predict equilibrium radiation, the temperature, density, and volume of the conical shock layer must be known. State properties were assumed to be constant in the shock layer. To locate the equilibrium shock-wave angle, the continuity equation for the mass of air swept out by the cone was solved simultaneously with the conservation equations for the inclined shock wave. This procedure is described in detail in appendix $B$ of reference 1 . The radiating volume was assumed to terminate with the first Mach line from the expansion fan at the base of the cone. (Radiation from the equilibrium expansion fan was also computed and found to be small.) A section through the radiating volume is shown in the following sketch, from which it can be.

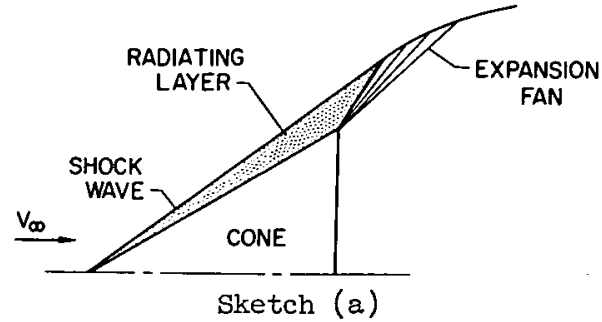

seen that the region behind the corner contributes significantly (approximately 20 percent) to the total volume. Equilibrium radiative intensity predictions were taken from reference 3 .

\section{Nonequilibrium Radiation}

At low free-stream densities substantial portions of the shock layer were out of equilibrium, and the radiation calculations were extended to include nonequilibrium effects. The experiments reported in reference 4 show that the nonequilibrium radiative intensity behind a normal shock wave has the form shown in Fig. 4(a) - a rapid rise to a peak followed by a relatively slow decay to the equilibrium level. Since the chemical properties and population of excited states in the nonequilibrium zone are primarily due to binary collisions, the peak of the radiation is approximately proportional to the first power of density, and the length of the nonequilibrium zone is approximately proportional to the inverse first power of density. These facts lead to the conclusion that, as long as the peak intensity is much greater than the equilibrium intensity, the heattransfer rate to a body placed at the termination of the nonequilibrium zone is nearly independent of density for a given free-stream velocity. This heat-transfer rate has been measured for some freestream velocities and is reported in references 4, 5 , and 6 . In addition, the excitation and relaxation distances (distances for the radiation to rise to its peak and to decay to 1.1 times the equilibrium value, respectively) have been reported in reference 4. For the purposes of the present computations, the radiation distribution shown in Fig. 4(a) has been approximated by the distribution shown in Fig. 4(b) - a linear rise to the peak value, followed by an exponential decay to the equilibrium level. This approximation gives the following equations for the radiative intensity behind a normal shock wave:

$E=E_{P} \frac{x^{*}}{\delta_{p}^{*}}$

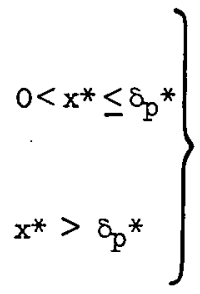

$\left.\mathrm{E}=\left(\mathrm{E}_{\mathrm{P}}-\mathrm{E}_{\mathrm{EQ}}\right) \mathrm{e}^{-\mathrm{k}^{*}\left(\mathrm{x}^{*}-\delta_{\mathrm{p}} *\right)}+\mathrm{E}_{\mathrm{EQ}} \mathrm{x}^{*}>\delta_{\mathrm{p}}^{*}\right)$

With the additional assumption that the peak radiation occurs at one tenth of the total distance to the point at which the radiation has decayed to $1.1 \mathrm{E}_{\mathrm{EQ}}$, that is, $\delta_{\mathrm{p}}^{*}=0.1 \delta_{\mathrm{r}}^{*}$ (ref. 4), integrating Eqs. (1) will give the heat-transfer rate from the nonequilibrium layer to a plane at the end of the relaxation zone.

$$
\begin{aligned}
\frac{W}{A}= & \int_{0}^{10 \delta_{\mathrm{p}}^{*}} \frac{\mathrm{E}}{2} d x^{*} \\
= & \frac{1}{2} \int_{0}^{\delta_{\mathrm{p}^{*}}} \mathrm{E}_{\mathrm{P}} \frac{x^{*}}{\delta_{\mathrm{p}^{*}}} d \mathrm{x}^{*} \\
& +\frac{1}{2} \int_{\delta_{\mathrm{p}^{*}}}^{10 \delta_{\mathrm{p}}^{*}}\left[\left(\mathrm{E}_{\mathrm{P}}-\mathrm{E}_{\mathrm{EQ}}\right) \mathrm{e}^{-\mathrm{k}^{*}\left(\mathrm{x}^{*}-\delta_{\left.\mathrm{p}^{*}\right)}+\mathrm{E}_{\mathrm{EQ}}\right] \mathrm{dx^{* }}}\right.
\end{aligned}
$$

Performing the integration gives 


$$
\frac{\mathrm{W}}{\mathrm{A}}=\frac{\mathrm{E}_{\mathrm{P} \mathrm{p}^{*}}}{4}+-\frac{9 \mathrm{E}_{\mathrm{EQ}}}{2} \delta_{\mathrm{p}^{*}}+\frac{\left(\mathrm{E}_{\mathrm{P}}-\mathrm{E}_{\mathrm{EQ}}\right)}{2 \mathrm{k}^{*}}\left(1-\mathrm{e}^{-\mathrm{g \textrm {k } ^ { * }} \delta_{\mathrm{p}}^{*}}\right)
$$

In Eq. (3) the unknowns are $\mathrm{k}^{*}$, the exponent controlling the decay rate, and $E_{P}$, the peak value of the radiative intensity.

A second equation relating $E_{P}$ and $k^{*}$ is obtained when Eqs. (1) are written for $x^{*}=\delta_{x^{*}}$

$$
1.1 \mathrm{E}_{\mathrm{EQ}}=\left(\mathrm{E}_{\mathrm{P}}-\mathrm{E}_{\mathrm{EQ}}\right) \mathrm{e}^{-9 \mathrm{k}^{*} \delta_{\mathrm{p}}^{*}}+\mathrm{E}_{\mathrm{EQ}}
$$

Solving Eqs. (3) and (4) simultaneously gives values for $E_{p}$ and $k^{*}$ as functions of density $\rho_{\infty}$. These equations are valid when $E_{P}$ is much greater than $E_{E Q}$, that is, at low densities. For higher densities, where this inequality no longer holds, $E_{\mathrm{P}} \rho_{\infty}$ was assumed constant in accord with binary scaling, and the value of $k^{*}$ was again computed from $\mathrm{Eq}$. (4). The results of the computations are show in table $I$ and apply to the streamlines behind a normal shock wave at a velocity of $4.0 \mathrm{~km} / \mathrm{sec}$. This velocity was chosen as the velocity normal to the conical swept shock wave for a $30^{\circ}$ half-angle cone at a free-stream velocity of $7 \mathrm{~km} / \mathrm{sec}$. Relaxation times were computed from the relaxation distances by assuming that the velocity behind the normal shock wave changed exponentially from the full frozen value inmediately behind the shock wave to 1.01 times the equilibrium level at the time when the radiant level had reached 1.1 times the equilibrium level.

To apply these results to cones, it was assumed that the relaxation times were the same in the conical layer as behind the normal shock wave for corresponding free-stream densities and shock strengths. Since the air velocity in the conical layer is much higher (approximately a factor of 10) than behind the normal shock wave, the air flows approximately 10 times farther along the cone before the radiation returns to its equilibrium level. Therefore, the excitation and relaxation distances and the exponential radiative intensity decay factors were adjusted to account for this high tangential velocity. The adjusted values for the conical shock layer are also given in table I. It should be noted that the radiant heat-transfer rate from the nonequilibrium zone to the surface of the cone is the same as the heat-transfer rate to a body placed at the termination of the nonequilibrium zone behind a normal shock wave in one-dimensional flow.

With the radiant intensity distribution along streamlines in the conical layer known, the total radiant output can be obtained by integrating the local radiant intensity over the volume of the shock layer. Two cases arise:

1. The shock layer is near equilibrium. For this case, the shock-wave shape was assumed to differ negligibly from a cone with the equilibrium shock angle. The integration then gave a closedform solution for the heat-transfer rate to the conical surface and for the total radiant output of the shock layer.

2. The shock layer is substantially out of equilibrium. For this case, the shock wave is no longer straight, and the shock-layer volume is larger than for equilibrium. The location of the shock wave was computed by assuming the density distribution along streamines to be exponential in the same manner as the velocity distribution described earlier. The integration of the radiant intensity was then performed numerically. Details of these procedures are described in appendix $B$.

A special problem arises when the density is low enough that the nonequilibrium radiative profile is severely truncated; that is, when the rediation from the streamline entering at the tip of the cone does not reach its peak value before the streamline reaches the base of the cone. When this occurs, the calculated radiative power becomes quite sensitive to the manner in which the radiation is assumed to rise to $E_{p}$. As can be seen from Fig. 4(a), the assumption of a straight line rise to $\mathrm{E}_{\mathrm{P}}$ may not be the best choice. In order to pick a rise with zero slope at $\delta_{\mathrm{p}}$, a quarter cycle of a sine wave was used. However, comparison with the actual rise histories from reference 4 show that the sine wave somewhat overpredicts the radiant intensity and thus will overpredict the total radiant power. Predictions for both a straight line rise and a quarter sine wave rise are shown in the final results.

The procedure described above is open to criticism on several points. First, it does not take into account any viscous effects; calculations by the methods described in reference 7 show that for the lowest test densities, the displacement thickness of the boundary layer is an appreciable fraction of the shock-layer thickness even for the nonablating models. The thick boundary layer will tend to push the shock wave away from the cone, effectively increasing the shock strength and thus increasing the radiant intensity. Furthermore, the high-temperature air in the boundary layer will contribute to the radiation. Second, there are no experiments to verify either the equilibrium radiative intensities predicted for these temperatures and densities or the nonequilibrium radiative heating rates, since the conditions for the present tests correspond to normal shock velocities below those previously studied. In fact, the nonequilibrium heating-rate data from reference 5 must be extrapolated from a velocity of 6 to $4 \mathrm{~km} / \mathrm{sec}$ and this extrapolation is certainly open to question. Third, no attempt was made to treat the expansion wave at the base of the cone under nonequilibrium conditions. These difficulties, along with the other approximations already noted, make the method approximate at best.

In spite of the disadvantages, the correlation of the predictions with the data is reasonable, as will be shown later. The method has advantages as well. It is relatively simple to use and does not require detailed computations of the local thermodynamic properties in the conical shock layer. It can be used to predict local radiant heat-transfer rates to the conical surface as well as the total radiant power in the conical shock layer.

\section{Results and Discussion}

The test results demonstrate experimentally the validity of the thesis of reference 1 - that radiative heat load is vastly smaller on cones than on comparable blunt bodies. In fact, the radiative heat lor d measured in the present tests on $30^{\circ}$ half-angle cones is 2 to 3 orders of magnitude less than the radiative heat load reported in reference 5 
on blunt bodies (when adjustments of the data from reference 5 are made to comparable test conditions and body size). This reduction in radiative heat load results directly from reduced shocklayer temperatures, which, in turn, result from substitution of oblique conical shock waves for strong detached shock waves.

The broadband radiometer data from the present tests are shown in Fig. 5, where the radiant power emitted from the shock layer in watts (corrected for the effects enumerated in the section on data reduction) is plotted as a function of the calculated equilibrium shock-layer density in amagats. Each point plotted on Fig. 5 represents an average of the data points obtained for that density and model material. Data are shown for the three model materials: polyethylene, metal, and polyformaldehyde. It can be seen from the figure that the radiation from the ablating polyethylene models is always greater - by factors of 1.7 to 4 - than that from the nonablating metal models and the ablating polyformaldehyde models. This is due to radiation from heated products of ablation in the boundary layer on the polyethylene models. Spectra from a polyethylene model and a metal model at identical free-stream conditions are shown in Fig. 6. It can be seen that the spectrum from the ablating polyethylene shows radiation in the visible and infrared which exceeds that in the spectrum from the nonablating metal model. The spectral range covered by the broadband detectors is also shown, and it is evident that it includes part of the radiation from ablation products. Thus the results shown in Fig. 5 for the polyethylene models are influenced by this extraneous source of radiation. Ablation-products radiation has been studied experimentally for blunt bodies in reference 2, where a method was developed for its correlation, and for cones in reference 7 , in which the correlation parameters from the blunt-body tests were found to predict successfully the measured ablation-products radiation on cones. Reference 7 also points out that radiation from the hot surface of the model is not sufficient to account for the observed radiation, as is demonstrated on Fig. 6 where the maximum possible surface contribution is shown multiplied by 100 for an assumed wall temperature of $1000^{\circ} \mathrm{K}$. It was not possible to obtain data on nonablating metal models at higher densities with the present equipment, since the extremely high heating rates at the sharp tip of the model would cause ablation to begin before the model reached the radiometer stations. Tests were therefore conducted with polyformaldehyde models for which the spectra (unpublished) from blunt-body tests indicated that radiation from ablation products would negligibly influence the broadband measurements. The resulting spectra on cones also showed that the radiation from ablation products in the range of the broadband detectors was small. The good agreement shown on Fig. 5 between the results obtained with the ablating polyformaldehyde models and the nonablating metal models at 0.25 amagat further supports this conclusion. Therefore the radiant power shown on Fig. 5 for the polyformaldehyde models is primarily from air.

Fig: 5 also shows the predictions for air radiation in the conical shock layer made by the methods described earlier. Predictions are shown for both equilibrium and combined equilibriumnonequilibrium radiation. The agreement between the measurements made on metal and polyformaldehyde models and the predictions which include both equilibrium and nonequilibrium effects is reasonably good. At the highest densities the measurements are approaching the equilibrium predictions. The measurements made on polyethylene models are substantially above the prediction at all densities, primarily because of the influence of ablationproducts radiation discussed earlier. However, it should be noted that at the lower test densities, viscous effects may also be important, since at these low densities the boundary-layer thickness is becoming a substantial fraction of the shocklayer thickness.

All of the data in Fig. 5 are presented as if the cones were at zero angle of attack. Since this was never actually the case, corrections were derived to account for the resulting change in radiant power. The method for deriving these corrections is presented in the section on data reduction and in appendix $\mathrm{A}$. If these corrections are successful, they should have two effects on the raw data. The first is to give the same deduced radiative power for each pair of radiometers placed at the same measuring station, but viewing the model from different directions; and the second is to make the deduced radiative power appear independent of angle of attack.

Typical results are shown in Fig. 7 for the polyethylene models at a free-stream density of $100 \mathrm{~m} \mathrm{Hg}$, where the radiant power is plotted as a function of angle of attack. (These data have already been corrected for slight velocity differences.) The circles show the data before the angle-of-attack correction was applied and the squares show the data after correction. Pairs of data points at the same $a$ are from simultaneous measurements. It can be seen that, for the most part, the corrected data have the desired behavior; indicating that the corrections are in fact reasonably successful in removing the angle-of-attack effects.

It will be noted in Fig. 7 that the uncorrected data do not continue to increase with increasing angle of attack. This is because the radiometer does not always view the model from the same direction (see sketch (c), appendix A). Thus the measured radiation depends on both angle of attack and radiometer viewing angle. The angleof -attack correction was derived to account for both of these effects.

After the corrections had been applied to the broadband data, considerable scatter existed for which causes could not be found. Root-mean-square deviations from the average values of radiant power are given in table II. No root-mean-square deviation is given for the polyethylene model data at a free-stream pressure of $2 \mathrm{~mm} \mathrm{Hg}$ because of Insufficient date.

\section{Conclusions}

From the results of these studies of radiant emission from the shock layer of conical models it can be concluded that: 
1. The measurements of radiation show that the substantial reduction in radiative heat load predicted for sharp cones over blunt bodies is, in fact; realized.

2. A method has been developed for predicting in some detall the radiative heat load and heattransfer rate to cones from air in the shock layer. The method accounts for both equilibrium and nonequilibrium radiation.

3. Measurements of radiation on nonablating metal models and ablating polyformaldehyde models compared with the predictions for air radiation show reasonable agreement.

4. Measurements of radiation on ablating polyethylene models show that the radiation from heated products of ablation in the boundary layer provides a net increase in heat load, as shown by comparison of data from the ablating polyethylene models with data from the nonablating metal models.

5. The effects of angle of attack on the data have been successfully correlated.

\section{Appendix A}

\section{Angle-of-Attack Corrections}

The following procedure was used to form a correction factor to account for the effect on the radiometer data of angle of attack of the model.

The coordinate system is shown in sketch (b) where $\varphi$ is the angle around the base of the cone, and $\varphi=0$ is at the ray on the cone surface which experiences the maximum inclination toward the freestream velocity vector.

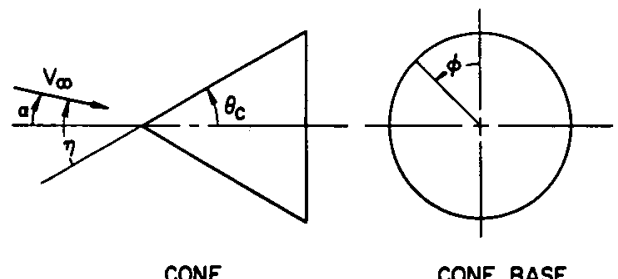

Sketch (b)

State properties in the shock layer, for a. given value of $\varphi$, were assumed to be constant along lines perpendicular to the conical surface and were calculated by the mass-flow method, described in the text, by assuming that they were the properties on the appropriate tangent cone. Thus, the distributions of temperature, density, and shock-wave angle were assumed to be functions only of the angle $\eta$ between rays on the surface of the cone and the velocity vector.

The relation between $\phi, \alpha$, and $\eta$ is

$\cos \eta=\cos \alpha \cos \theta_{c}-\cos \phi \sin \alpha \sin \theta_{c}$

With temperature, density, and shock-wave angle known as functions of $\varphi$ and $\alpha$, the ratio $E_{E Q} /\left(E_{E Q}\right)_{\alpha=0}$ was computed as a function of $\varphi$ and $\alpha$ by use of the equilibrium predictions of reference 3 . It was found that, for the range of free-stream densities for which the shock layer was near equilibrium, this ratio was nearly independent of free-stream density. The ratio $V_{E Q} /\left(V_{E Q}\right)_{\alpha=0}$ was also computed. This ratio is the relative increase or decrease in local shock-layer volume as a function of $\varphi$ and $\alpha$ and was also found to be nearly independent of free-stream density.

These two ratios were combined to give $\mathrm{E}_{\mathrm{EQ}} \mathrm{V}_{\mathrm{EQ}} /\left(\mathrm{E}_{\mathrm{EQ}} \mathrm{V}_{\mathrm{EQ}}\right)_{\alpha=0}$. This ratio represents the local value of the radiation in watts as a function of $\phi$ and $\alpha$, divided by the value at $\alpha=0$. To form a correction factor it was integrated over the portion of the conical shock layer visible to the photomultiplier. For this purpose it was assumed that the photomultiplier viewed 64 percent of the volume before the shoulder of the model (this percentage was determined graphically) and all of the volume between the shoulder and the first wave of the expansion fan. Computations of the total radiating volume showed that 80 percent of the volume was in front of the shoulder and 20 percent was between the shoulder and the expansion fan. given by

The angle-of-attack correction is therefore

$$
\begin{aligned}
x\left(\sigma_{\mathrm{V}}, \alpha\right)=\frac{\mathrm{W}}{\mathrm{W}_{\alpha=0}}= & \frac{0.8 \int \frac{E_{E Q} \mathrm{~V}_{E Q}}{\left(E_{E Q} \mathrm{~V}_{E Q}\right)_{\alpha=0}} \mathrm{d \varphi}}{0.64(2 \pi)} \\
& +\frac{0.2 \int_{0}^{2 \pi} \frac{E_{E Q} V_{E Q}}{\left(E_{E Q} V_{E Q}\right)_{\alpha=0}} d \varphi}{2 \pi}
\end{aligned}
$$

where the first term on the right accounts for the volume before the shoulder and was integrated over the portion of the cone visible to the photomultiplier. The second term accounts for the volume between the shoulder and the expansion fan and was integrated completely around the cone. The viewing angle, $\sigma_{\mathrm{V}}$, which defines the direction from which the photomultiplier views the model, is shown in sketch (c).

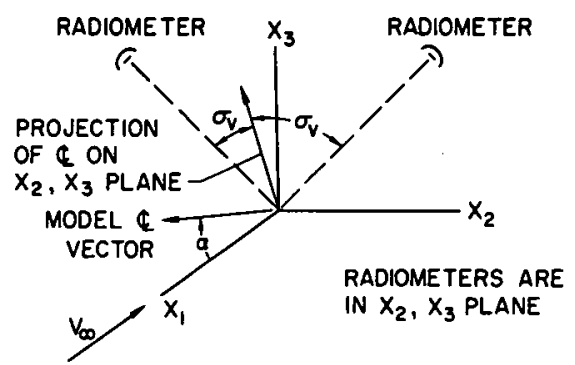

Sketch (c)

Figure $8(a)$ shows the values of $x$ plotted as a function of viewing angle for various angles of attack. The corrections shown are for equilibrium and, when applied to the raw data, it was apparent that, at the lower test densities, the corrections were too large. Since the radiation at these lower densities was known to be primarily nonequilibrium, the corrections were recalculated with the nonequilibrium radiation measurements from 
reference 5 substituted for the equilibrium values. The computed corrections are shown in Fig. 8(b) to be considerably smaller than the corrections for equilibrium. This is because the dependence of radiation on velocity normal to the shock wave is much less for nonequilibrium than for equilibrium. When the corrections for nonequilibrium were applied to the test data at low densities, the results were adequate. Equilibrium corrections were used for free-stream pressures of 100 and $200 \mathrm{~mm} \mathrm{Hg}$, and nonequilibrium corrections were used for pressures of $50 \mathrm{~mm} \mathrm{Hg}$ and below.

It is evident that a straightforward extension of this method could be used to obtain estimates of the local heat-transfer rates and total radiant heat load on cones flying at angle of attack.

A better approach would be to treat each data point individually, by dividing the shock layer into a number of streamtubes and computing the radiation from each streamtube separately, taking into account the distribution of radiation along each streamtube. However, this approach appeared to be prohibitively time consuming and, in view of the reasonable results obtained with the simpler methods, unnecessary.

\section{Appendix B}

Integration of Radiation in the Conical Shock Layer

The text describes the models of radiation used to obtain the radiation distribution from air along streamlines in the conical shock layer. In order to obtain estimates of the total radiant power from these distributions, it was necessary to perform a volume integral of the local radiant intensity over the radiating volume.

The coordinate system used for this purpose is shown in the sketch below

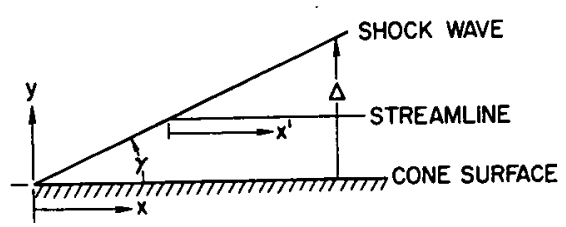

Sketch (d)

The shock standoff distance is

$$
\Delta=x \tan \gamma
$$

For purposes of this integration, streamlines were assumed to be straight lines parallel to the body surface. The distance along a streamline measured from the shock wave is given by $x^{\prime}$. The $y$ coordinate of a streamline is constant and is given by

$$
y=\left(x-x^{\prime}\right) \tan \gamma
$$

Combining Eqs. (BI) and (B2) and rearranging gives

$$
\mathrm{x}^{\prime}=\frac{\Delta-\mathrm{y}}{\tan \gamma}
$$

The distribution of radiation along streamines is given by Eqs. (1) of the text, with the notation changed to that for streamines in the conical shock layer, as

$$
\left.\begin{array}{ll}
E\left(x^{\prime}\right)=E_{P} \frac{x^{\prime}}{\delta_{p}} & 0<x^{\prime} \leq \delta_{p} \\
E\left(x^{\prime}\right)=\left(E_{P}-E_{E Q}\right) e^{-k\left(x^{\prime}-\delta_{p}\right)}+E_{E Q} & x^{\prime}>\delta_{p}
\end{array}\right\}
$$

Making the change of variables in Eqs. (B4) to the cone-apex-oriented coordinate system described by Eqs. (BI), (B2), and (B3) gives for the distribution of radiation in the contcal shock layer

$$
\left.\begin{array}{l}
\mathrm{E}(\mathrm{x}, \mathrm{y})=\mathrm{E}_{\mathrm{P}} \frac{\frac{\Delta-\mathrm{y}}{\tan \gamma}}{\delta_{\mathrm{p}}} \quad \mathrm{y}_{\mathrm{p}}=\left(\Delta-\delta_{\mathrm{p}} \tan \gamma\right) \leq \mathrm{y}<\Delta \\
\mathrm{E}(\mathrm{x}, \mathrm{y})=\left(\mathrm{E}_{\mathrm{P}}-\mathrm{E}_{\mathrm{EQ}}\right) \mathrm{e}^{-\mathrm{k}\left(\frac{\Delta-\mathrm{y}}{\tan \gamma}-\delta_{\mathrm{p}}\right)}+\mathrm{E}_{\mathrm{EQ}} \quad \mathrm{y}<\mathrm{y}_{\mathrm{p}}
\end{array}\right\}
$$

Integrating first with respect to y gives the infinite, thin, plane shock-layer idealization to the local heat-transfer rate. The integrals are

$$
\begin{aligned}
& \frac{W}{A}=\int_{0}^{\Delta} \frac{E_{P}}{\delta_{p}}\left(\frac{\Delta-y}{\tan \gamma}\right) d y \quad 0<x \leq \delta_{p}
\end{aligned}
$$

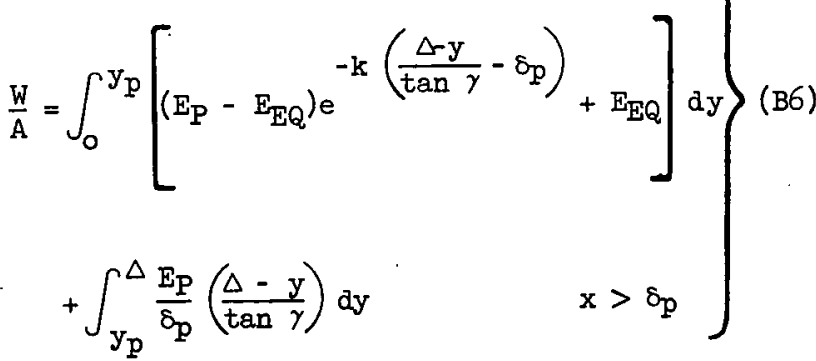

where W/A is twice the loc日l heat-transfer rate and the area $A$ is a function of $x$. This factor of 2 occurs since the equations in this appendix are written for the total radiant emission from the shock layer, of which only about 50 percent is incident on the surface. Integrating Eqs. (B6) gives

$$
\left.\begin{array}{rl}
\frac{W}{A \tan \gamma}= & \frac{E_{\mathrm{P}}}{2} \frac{x^{2}}{\delta_{p}} \\
\frac{W}{A \tan \gamma}=E_{E Q}\left(x-\delta_{p}\right)-\frac{E_{P}-E_{E Q}}{k}\left[e^{-k\left(x-\delta_{p}\right)}-1\right] \\
+\frac{E_{P}}{2} \delta_{p} & 0<x \leq \delta_{\mathrm{p}} \\
& x>\delta_{p}
\end{array}\right\}(B 7)
$$

Integrating once more, over the surface area of the cone, will give the total radiant power. The surface area element is given by

$$
d A=2 \pi x \sin \theta_{c} d x
$$

where $\theta_{\mathrm{c}}$ is the half apex angle of the cone. 
Thus the power can be written

$$
\left.\begin{array}{rl}
\mathrm{W}= & 2 \pi \tan \gamma \sin \theta_{\mathrm{c}} \int_{0}^{l} \frac{\mathrm{E}_{\mathrm{p}}}{2} \frac{\mathrm{x}^{3}}{\delta_{\mathrm{p}}} \mathrm{dx} \quad \imath \leq \delta_{\mathrm{p}} \\
\mathrm{W}= & 2 \pi \tan \gamma \sin \theta_{\mathrm{c}}\left(\int_{0}^{\delta_{\mathrm{p}}} \frac{\mathrm{E}_{\mathrm{P}}}{2} \frac{\mathrm{x}^{3}}{\delta_{\mathrm{p}}} \mathrm{dx}\right. \\
& +\int_{\delta_{\mathrm{p}}}^{l} \int_{\mathrm{E}_{\mathrm{EQ}}\left(\mathrm{x}^{2}-\mathrm{x} \delta_{\mathrm{p}}\right)} \\
& +\frac{\mathrm{E}_{\mathrm{P}}-\mathrm{E}_{\mathrm{EQ}}}{\mathrm{k}} \mathrm{x}\left[\mathrm{e}^{-\mathrm{k}\left(\mathrm{x}-\delta_{\mathrm{p}}\right)}-1\right] \\
& \left.+\frac{\left.\left.\mathrm{E}_{\mathrm{P}} \mathrm{x} \delta_{\mathrm{p}}\right\} \mathrm{dx}\right)}{2}\right)
\end{array}\right\}
$$

It is convenient to normalize Eqs. (B9) with respect to the total radiation that would be obtained if the shock layer were fully in equilibrium. For equilibrium, the radiation along streamlines is constant and twice the heat-transfer rate is given by

$$
\frac{W_{E Q}}{A}=E_{E Q} \triangle
$$

The total equilibrium radiative power is given by the integration of $\mathrm{Eq}$. (BlO) with respect to $\mathrm{x}$ to give

$$
\mathrm{W}_{\mathrm{EQ}}=2 \pi \tan \gamma \sin \theta_{\mathrm{c}} \int_{0}^{2} \mathrm{E}_{\mathrm{EQ}} \mathrm{x}^{2} \mathrm{dx}
$$

Integrating Eqs. (B9) and (Bll) and taking the ratio $W / W_{E Q}$ gives finally

$$
\begin{aligned}
& \frac{\mathrm{W}}{\mathrm{W}_{\mathrm{EQ}}}=\frac{3}{8} \frac{\mathrm{E}_{\mathrm{P}}}{\mathrm{E}_{\mathrm{EQ}}} \frac{2}{\delta_{\mathrm{p}}} \\
& \imath \leq \delta_{\mathrm{p}} \\
& \frac{W}{W_{\mathrm{EQ}}}=\frac{3}{8} \frac{E_{\mathrm{P}}}{E_{\mathrm{EQ}}}\left[2 \frac{\delta_{\mathrm{p}}}{l}-\left(\frac{\delta_{\mathrm{p}}}{l}\right)^{3}\right] \\
& +\left[1-\frac{3}{2} \frac{\delta_{p}}{2}+\frac{1}{2}\left(\frac{\delta_{p}}{2}\right)^{3}\right] \\
& +3\left(\frac{E_{\mathrm{P}}}{\mathrm{E}_{\mathrm{EQ}}}-1\right)\left\{\frac{1}{2 \mathrm{k} l}\left[\mathrm{I}-\left(\frac{\delta_{\mathrm{p}}}{2}\right)^{2}\right]\right. \\
& +\frac{1}{(k l)^{3}}\left[(k l+1) e^{-k\left(l-\delta_{p}\right)}\right. \\
& \left.-\left(k \delta_{p}+1\right) !\right\} \\
& \imath>\delta_{\mathrm{p}}
\end{aligned}
$$

Equations (B12) give the radiant power in the conical shock layer for the volume ahead of the cone base. They do not include the power in the volume behind the cone base and before the expansion fan. (See sketch (a) for the definition of radiating volume.) To partially compensate for this difficulty, the value of $W_{E Q}$ for the total radiating volume was used in applying equation ( $\mathrm{BI2}$ ) to compute the radiation expected in the present tests. This assumes that the ratio $\mathrm{W} / \mathrm{W}_{\mathrm{EQ}}$ is the same for both volumes - an assumption which is not exact. However, the error resulting from this procedure is small since the volume behind the cone base comprises only 20 percent of the total volume.

Two other effects have been considered. The first of these occurs since, for nonequilibrium flow, the shock wave is curved and lies outside the equilibrium wave. Thus the integration procedure described above, which is over only the equilibrium volume, will underestimate the total radiative power. The effect will be largest at the lower densities. To account for this effect, the density was assumed to rise exponentially (along streamlines) from its frozen value immediately behind the shock wave to 0.99 of its equilibrium value at the point on the streamline where the radiation has decayed to $1.1 \mathrm{E}_{\mathrm{EQ}}$. The position of the shock wave was located by the mass-flow method described in the text for the nonconstant density. The equations for the total radiant power were then integrated numerically. It was found that the effect was small for free-stream pressures greater than $20 \mathrm{~mm} \mathrm{Hg}$. This effect has been included in the predictions presented in Fig. 5.

The second effect is described in the text. A sine wave was substituted for the initial linear rise of the radiation to $\mathrm{E}_{\mathrm{P}}$. The results are also shown in Fig. 5 .

\section{$\underline{\text { References }}$}

1. Allen, H. J., Seiff, A., and Winovich, W.: Aerodynamic Heating of Conical Entry Vehicles at Speeds in Excess of Earth Parabolic Speed. NASA TR R-185, 1963 .

2. Craig, Roger A., and Davy, William C.: Thermal Radiation From Ablation Products Injected Into a Hypersonic Shock Layer. NASA TN D-1978, 1963.

3. Kivel, B., and Bailey, K.: Tables of Radiation From High Temperature Air. Res. Rep. 2l, Ávco Res. Lab., 1957.

4. Teare, J. D., Georgiev, S., and Allen, R. A.: Radiation From the Nonequilibrium Shock Front. Res. Rep. 112, Avco-Everett Res. Lab., 1961.

5. Page, William A., and Arnold, James 0.: ShockLayer Radiation of Blunt Bodies at Reentry Velocities. NASA TR R-193, 1964.

6. Canm, J. C., Kivel, B., Taylor, R. L., and Teare, J. D.: Absolute Intensity of Nonequi.librium Radiation in Air and Stagnation Heating at High Altitudes. Res. Rep. 93, Avco-Everett Res. Lab., 1959.

7. Davy, William C., Craig, Poger A., Chapman, Gary T., and Compton, Dale L.: Ablation Product Radiation From Cones. AIAA Preprint 64-71, 1964. 
TABLE I. - CONSTANTS USED IN RADIATION CALCULATIONS FOR TEST CONDITIONS

\begin{tabular}{|c|c|c|c|c|c|c|}
\hline \multirow[b]{2}{*}{$\begin{array}{l}\mathrm{P}_{\infty}, \\
\min \mathrm{Hg}\end{array}$} & \multirow[b]{2}{*}{$\underset{\text { watts } / \mathrm{cm}^{3}}{\mathrm{E}_{\mathrm{P}}}$} & \multirow[b]{2}{*}{$\begin{array}{c}\mathrm{E}_{\mathrm{EQ}} \\
\text { watts }\end{array}$} & \multicolumn{2}{|c|}{$\begin{array}{c}\text { Normal shock } \\
\text { wave }\end{array}$} & \multicolumn{2}{|c|}{$\begin{array}{c}\text { Conical shock } \\
\text { layer }\end{array}$} \\
\hline & & & $\mathrm{cm}^{*}$ & $\begin{array}{l}\delta_{\mathrm{p}}^{*} \\
\mathrm{~cm}\end{array}$ & $\mathrm{~cm}^{-1}$ & $\begin{array}{l}\delta_{p} \\
\text { cm }\end{array}$ \\
\hline 5 & 1.77 & 0.176 & 3.02 & 0.0182 & 0.235 & 0.234 \\
\hline 10 & $3 \cdot 20$ & .297 & 5.58 & .00912 & .435 & .117 \\
\hline 20 & 4.55 & .970 & 8.80 & .00456 & .684 & .0586 \\
\hline 50 & 15.9 & 4.50 & 19.7 & .00182 & 1.54 & .0234 \\
\hline 100 & 31.5 & $13 \cdot 5$ & 31.6 & .000912 & 2.46 & .0117 \\
\hline 200 & 63.0 & 40.5 & 41.8 & .000456 & 3.25 & .00586 \\
\hline
\end{tabular}

TABLE II. - ROOT-MEAN-SQUARE DEVIATION OF BROADBAND RADIOMETER DATA

\begin{tabular}{|c|c|c|c|c|}
\hline $\begin{array}{c}\text { Model } \\
\text { material }\end{array}$ & $\begin{array}{c}\text { Free-stream } \\
\text { pressure, } \\
\text { mm Hg }\end{array}$ & $\begin{array}{c}\text { Average watts } \\
\text { observed } \\
\text { (Fig. 5) }\end{array}$ & $\begin{array}{c}\text { Number of } \\
\text { data points }\end{array}$ & $\begin{array}{c}\text { Root-mean-square } \\
\text { deviation, } \\
\text { watts }\end{array}$ \\
\hline Polyethylene & 200 & 4.1 & 10 & \pm 0.62 \\
& 50 & 3.9 & 12 & \pm .59 \\
& 20 & .58 & 10 & \pm .53 \\
& 10 & .34 & 8 & \pm .12 \\
& 5 & .18 & 11 & \pm .12 \\
& 200 & 2.0 & 14 & \pm .045 \\
\hline Polyformaldehyde & 100 & 1.0 & 12 & \pm .26 \\
& 50 & .71 & 10 & \pm .15 \\
& 20 & .31 & 2 & \pm .21 \\
& 20 & .36 & 23 & \pm .084 \\
\hline Metal & 10 & .21 & 8 & \pm .13 \\
& & & & \pm .063 \\
\hline
\end{tabular}



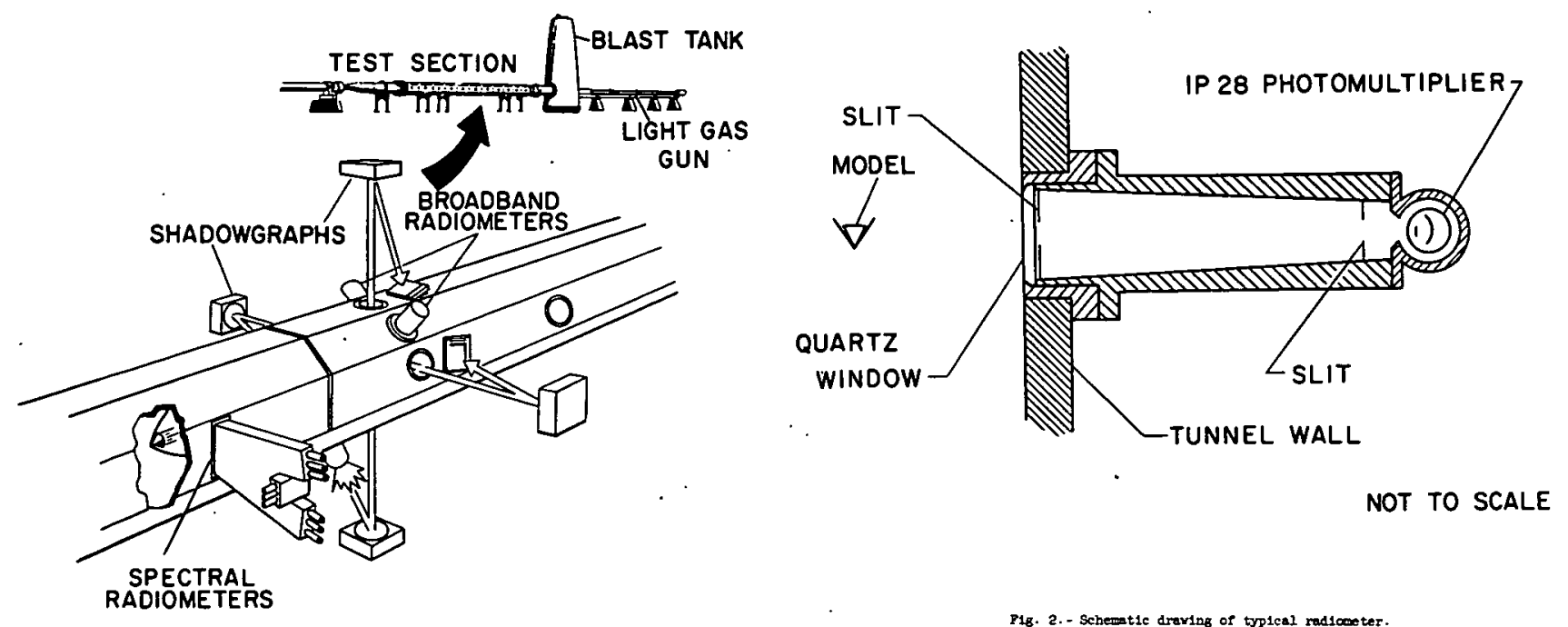

METERS

718. 1.- Schematic drewing of prototype bypervelocity ree-f1leht fac1lity.
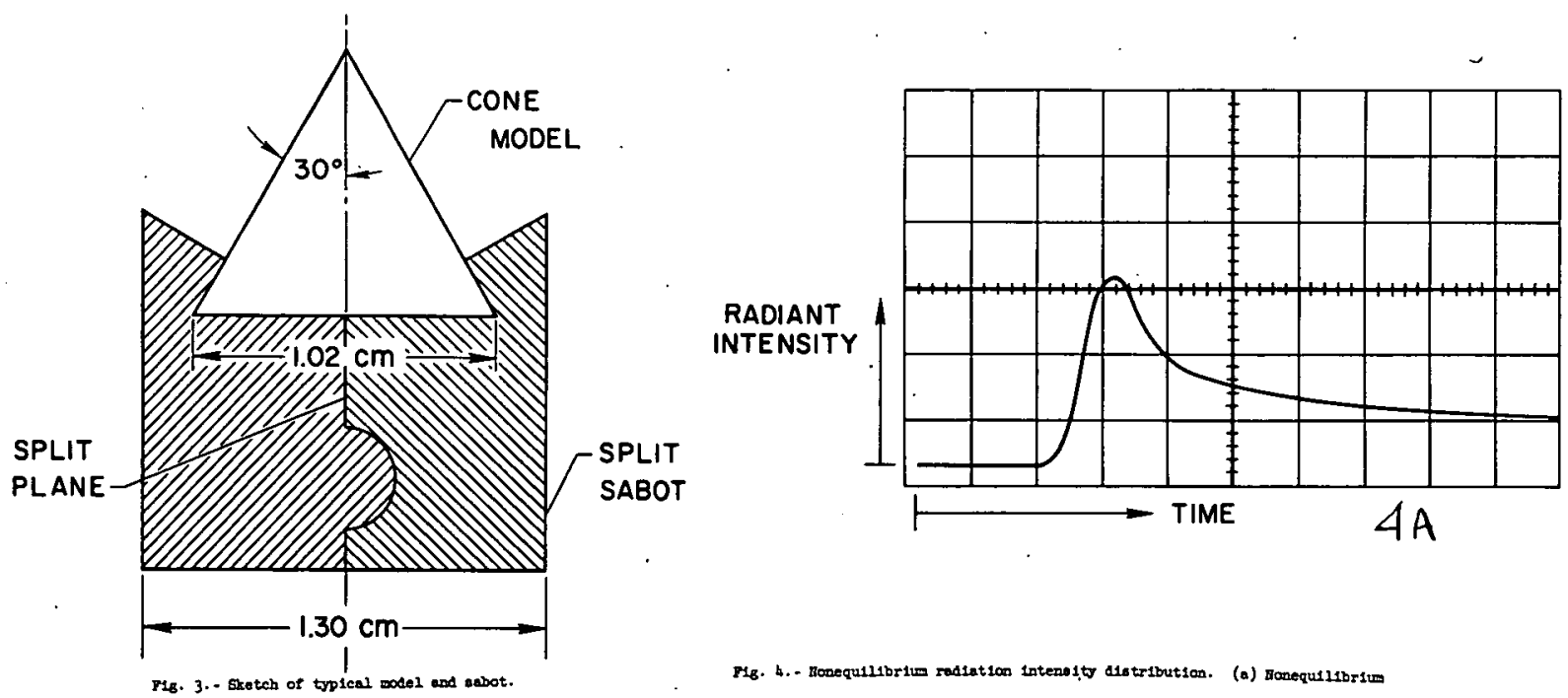

Fig. 3. - Sketch of topical wodel and abbot.

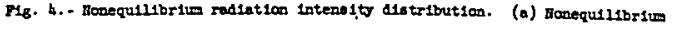
rediotion intens 1ty d1otribution behind a normal abock (rroo res. b.)

(b) Approximation to nonequilibrium raliation Intensity distribution used

for present computations.

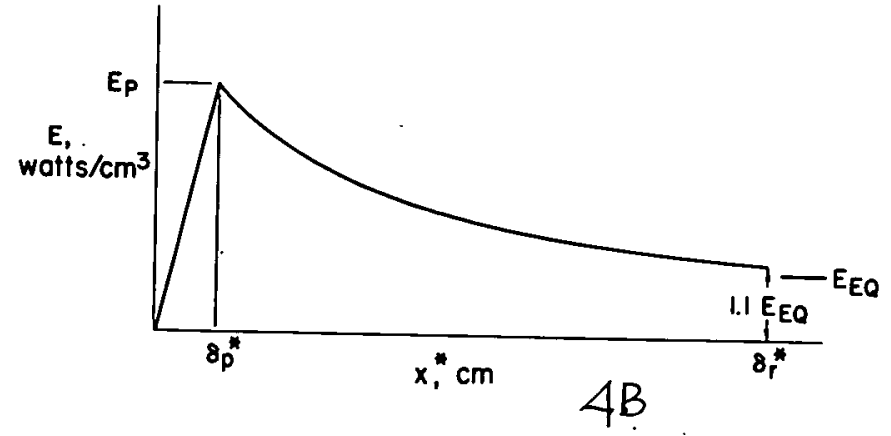




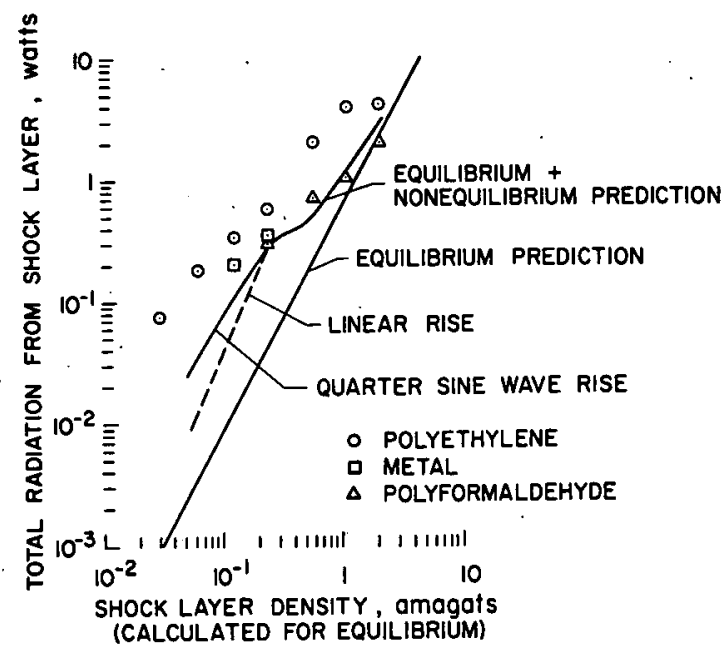

Fig. 5. - Measurements and predictions of total radiant porres for $1.02 \mathrm{~cm}$ base diameter, $30^{\circ}$ half-argle cones; $v_{\infty}=7.0 \mathrm{~km} / \mathrm{sec}$

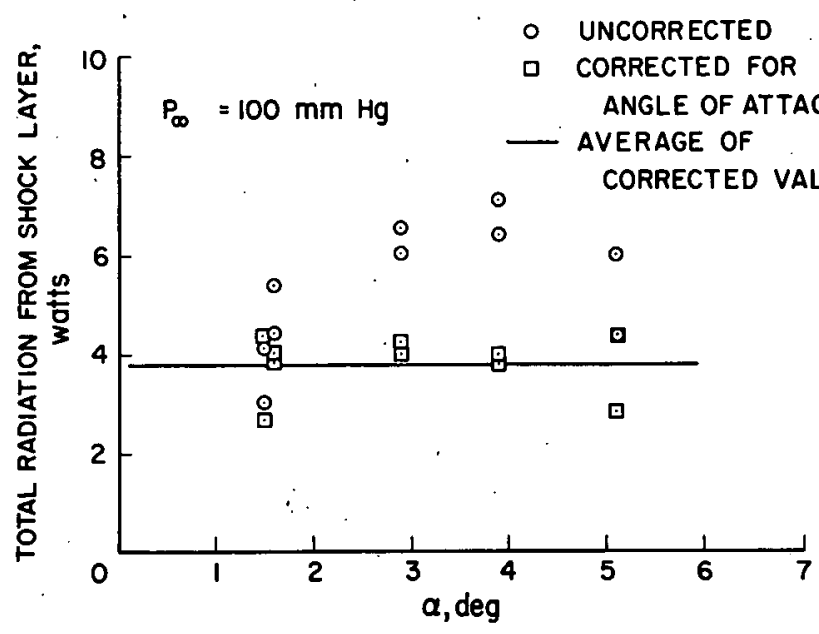

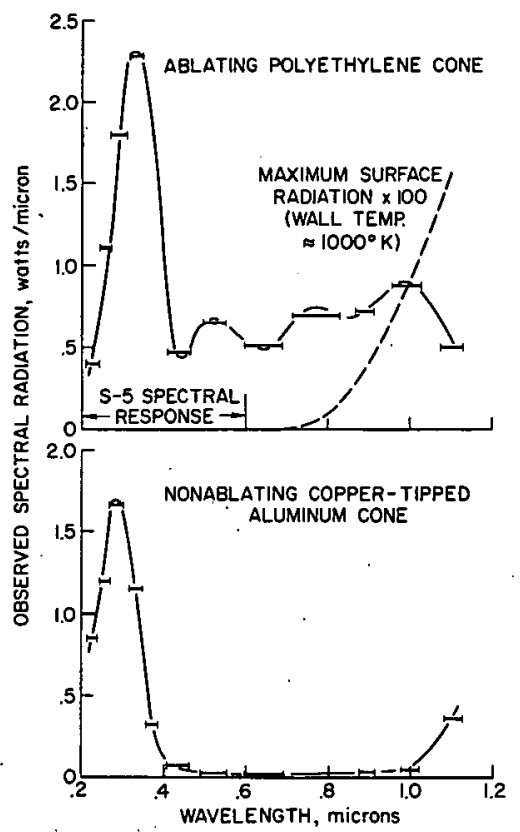

F1g. 6. - bxamples of spectra; $v_{\infty}=7.0 \mathrm{~km} / \mathrm{sec}, p_{\infty}=10 \mathrm{~mm}$ Hg.

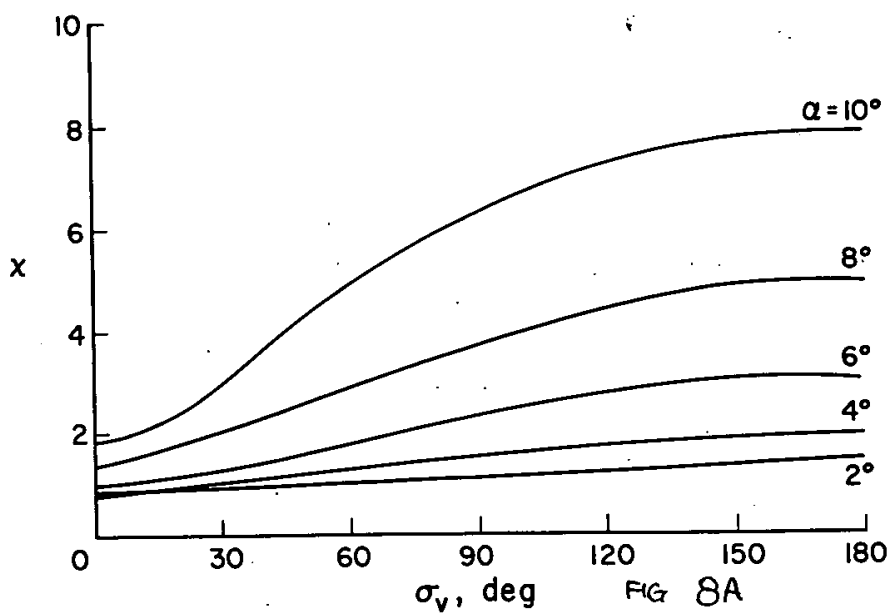

Fig. 7.. Typical effect of angle-of -attack correction on data; $V_{\infty}=7.0 \mathrm{ks} / \mathrm{sec}$

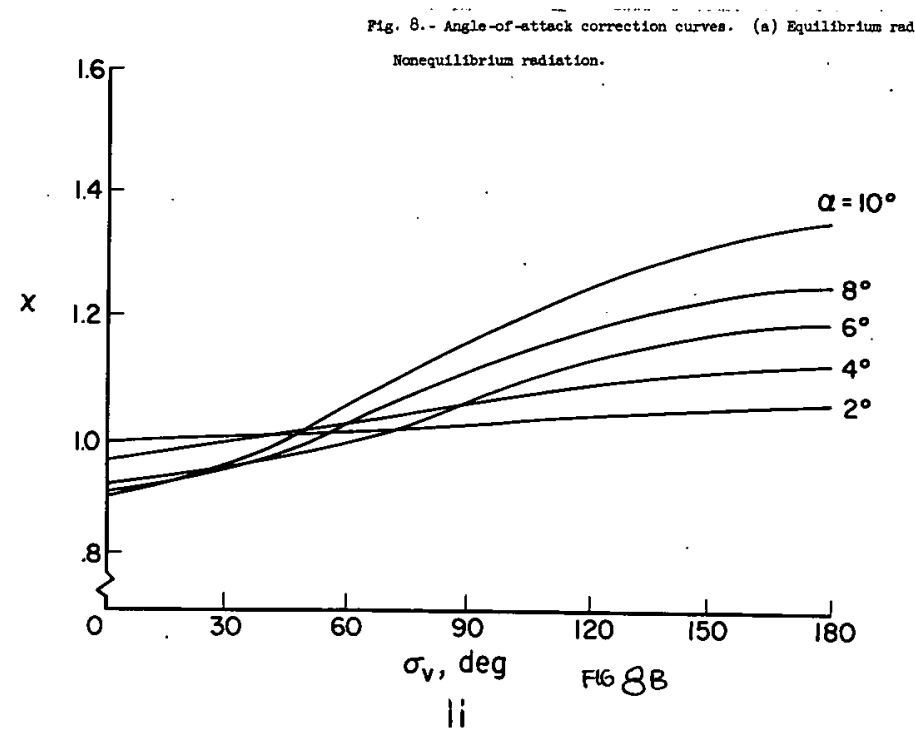

